\title{
Multiple Splenic Abscesses in a Case of Enteric Fever: Salvaging Spleen through Pigtail Drainage and Antibiotics is a Good Alternative Approach
}

\author{
Vijoy Kumar Jha, Tushar Vidhale ${ }^{1}$ \\ Departments of Nephrology and ${ }^{~}$ Medicine, Command Hospital Air Force, Bengaluru, Karnataka, India
}

\section{Abstract}

Splenic abscess is a rare complication of enteric fever in developing countries. This is seen mostly solitary rather than being multiple and can be fatal if untreated. In general clinical practice, multiple splenic abscesses or thick pus with septations may require splenectomy. Here, we report a rare case of enteric fever with multiple splenic abscesses in a young immunocompetent male who was successfully managed with antibiotics and percutaneous pigtail drainage of pus from the larger cavity.

Keywords: Antibiotics, percutaneous pigtail drainage, splenectomy, splenic abscess

\section{INTRODUCTION}

Splenic abscess is an uncommon disease, and about $70 \%$ develop in patients with concurrent infections. ${ }^{[1]}$ This is seen mostly solitary rather than being multiple and can be fatal if get unrecognized at an initial stage. ${ }^{[2]}$ Patients with multiple abscesses not responding to percutaneous drainage require splenectomy. We report a rare case of enteric fever with multiple splenic abscesses in a young immunocompetent male who was successfully managed with antibiotics and percutaneous pigtail drainage of pus from the larger cavity.

\section{Case Report}

$36 \mathrm{yr}$ old male, MK was admitted in our hospital with history of fever for 5 days - intermittent, moderate to high grade associated with chills and severe non colicky pain in left hypochondriac region of 3 days duration. Clinical examination revealed fever (temperature $-103^{\circ} \mathrm{F}$ ), tachycardia (pulse - 112/min, regular), normal blood pressure, and mild hepatosplenomegaly with left upper-quadrant tenderness. Initial evaluation revealed normal complete blood count with normal coagulation profile. Peripheral blood smear was suggestive of normocytic normochromic picture with no features of sepsis. Other investigations revealed normal liver

\begin{tabular}{|l|l|}
\hline \multicolumn{2}{|c|}{ Access this article online } \\
\hline Quick Response Code: & Website: \\
\hline & www.ijccm.org \\
\hline & \\
\hline
\end{tabular}

function test and renal function test, blood sugar profile was normal, and serology for malaria, dengue, and leptospira was negative. Blood culture grew Salmonella enterica spp. enterica serotype typhi which was sensitive to amikacin, ceftriaxone, piperacillin tazobactum, and meropenem. Typhi Dot-IgG and IgM both were positive. Urine/stool routine and microscopic examination was normal and culture sensitivity was sterile. Chest X-ray posteroanterior view was suggestive of mild left pleural effusion confirmed on ultrasonography (USG). Diagnostic pleural fluid analysis was lymphocyte predominant and exudative. Pleural fluid adenosine deaminase was normal and staining (fungal/Gram/acid-fast bacteria) was negative. USG abdomen revealed multiple (3-5) ill-defined hypo-echoic lesions in splenic parenchyma, largest $6.4 \mathrm{~cm} \times 2.5 \mathrm{~cm} \times 4 \mathrm{~cm}$ (approx. volume -35 cc). Contrast-enhanced computed tomography (CECT) abdomen done confirmed these findings [Figure 1]. Two-dimensional echo, hemoglobin electrophoresis, and

Address for correspondence: Dr. Vijoy Kumar Jha, Physician and Nephrologist, Department of Nephrology,
Command Hospital Air Force, Bengaluru - 560007 , Karnataka, India. E-mail: vkjhamd@gmail.com

This is an open access journal, and articles are distributed under the terms of the Creative Commons Attribution-NonCommercial-ShareAlike 4.0 License, which allows others to remix, tweak, and build upon the work non-commercially, as long as appropriate credit is given and the new creations are licensed under the identical terms.

For reprints contact: reprints@medknow.com

How to cite this article: Jha VK, Vidhale T. Multiple splenic abscesses in a case of enteric fever: Salvaging spleen through pigtail drainage and antibiotics is a good alternative approach. Indian J Crit Care Med 2018;22:886-8. 
viral markers (HIV, HBsAg, hepatitis C virus) were normal. He was put on injection ceftriaxone + amikacin for 14 days. He became afebrile after 5 days of intravenous (IV) antibiotics and remained afebrile for 1 week, but thereafter started having intermittent fever with mild left hypochondrium pain. His repeat blood culture was sterile. Peripheral blood smear was suggestive of neutrophilic leukocytosis (total leukocyte count $-14,000 / \mathrm{mm}^{3}$ and differential leukocyte count - N86) with mild shift to left. Other biochemical parameters were normal. His repeat USG abdomen revealed multiple hypoechoic lesions within spleen, with the largest dimension of $5.33 \mathrm{~cm} \times 5.62 \mathrm{~cm} \times 6.83 \mathrm{~cm}$. CECT abdomen done thereafter revealed multiple irregular hypodense splenic lesions, the largest measuring $96 \mathrm{~mm} \times 60 \mathrm{~mm} \times 53 \mathrm{~mm}$ and pleural effusion with collapse-consolidation part of the left lower lobe [Figure 2]. Mantoux test was negative, and serum angiotensin-converting enzyme was normal. In view of persistent fever and multiple enlarging abscesses despite antibiotics, the option of splenectomy was considered with gastrointestinal surgeon and the same was discussed with the relatives. However, considering his age and long-term consequences of splenectomy, we decided to adopt a spleen-conserving approach (with the option of

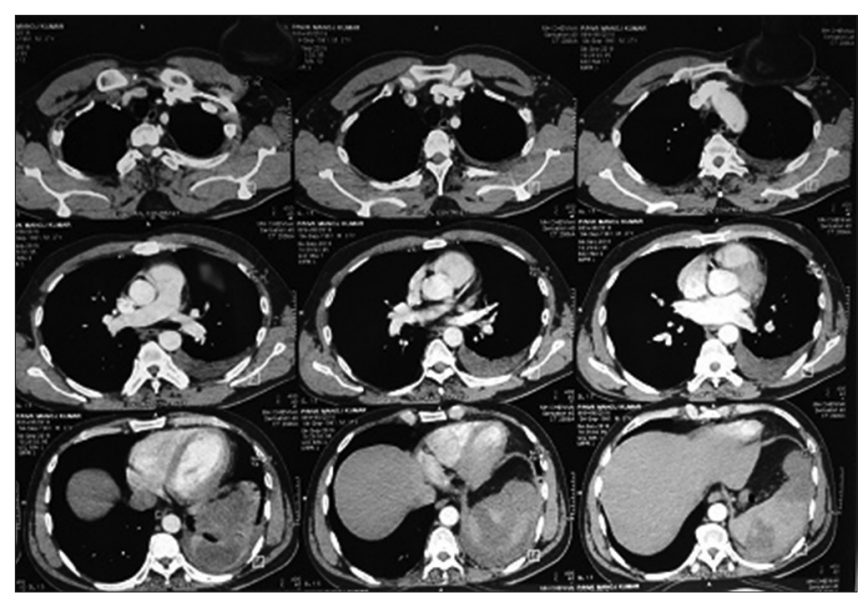

Figure 1: Contrast-enhanced computed tomography abdomen on initial presentation - multiple hypodense lesions (18-25 HU) scattered throughout spleen, predominantly peripherally based

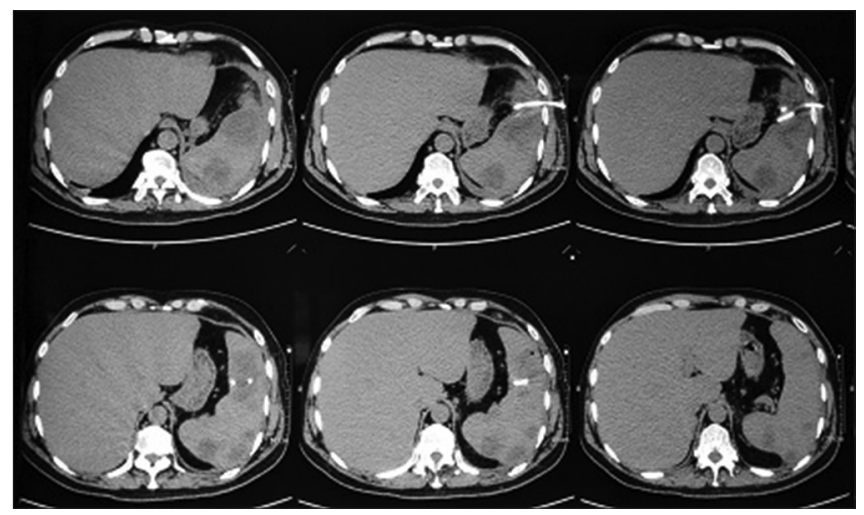

Figure 3: Contrast-enhanced computed tomography abdomen (Axial section) after putting pigtail drainage emergency splenectomy if required). Percutaneous pigtail drainage in larger cavity was put under USG guidance and $200-\mathrm{ml}$ pus was drained on the $1^{\text {st }}$ day [Figures 3 and 4]. The pigtail was removed after 5 days when the drainage was around 4-5 $\mathrm{ml}$. Antibiotics were changed to IV meropenem + clindamycin. The patient now responded well to antibiotics and US-guided percutaneous pigtail drainage. He became afebrile after 3 days of putting drainage. Pigtail drainage culture/sensitivity was sterile, and cytology revealed necrotic debris. He was discharged in a stable condition after 2 weeks of changed IV antibiotics course. On subsequent follow-up, he remained asymptomatic and the US abdomen has shown no residual abscess after 3 months.

\section{Discussion}

The clinical diagnosis of splenic abscess due to enteric fever is very difficult to make because of its rarity,

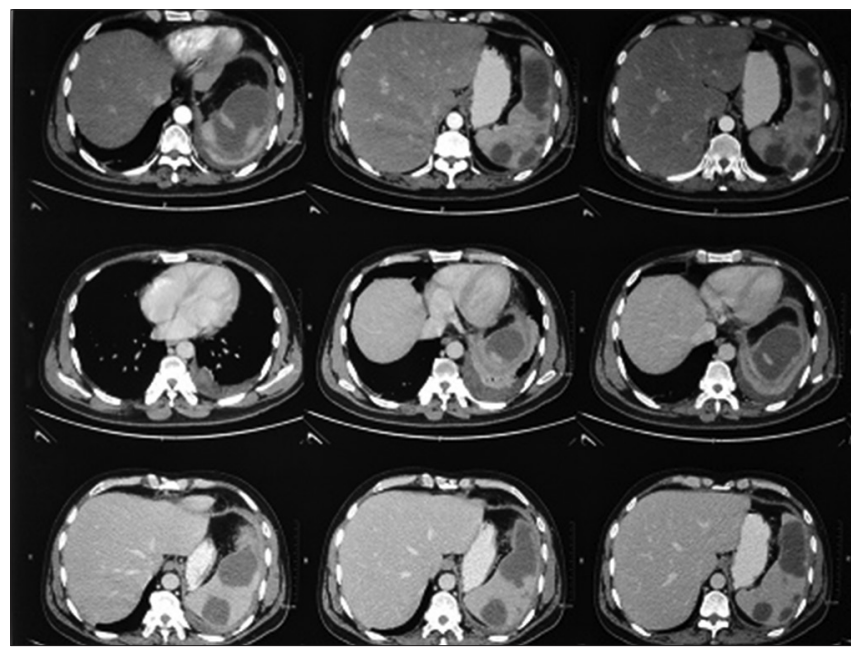

Figure 2: Contrast-enhanced computed tomography abdomen after 2 weeks - multiple irregular hypodense splenic lesions (20-24 HU), largest measuring $96 \mathrm{~mm} \times 60 \mathrm{~mm} \times 53 \mathrm{~mm}$. Other lesions are smaller

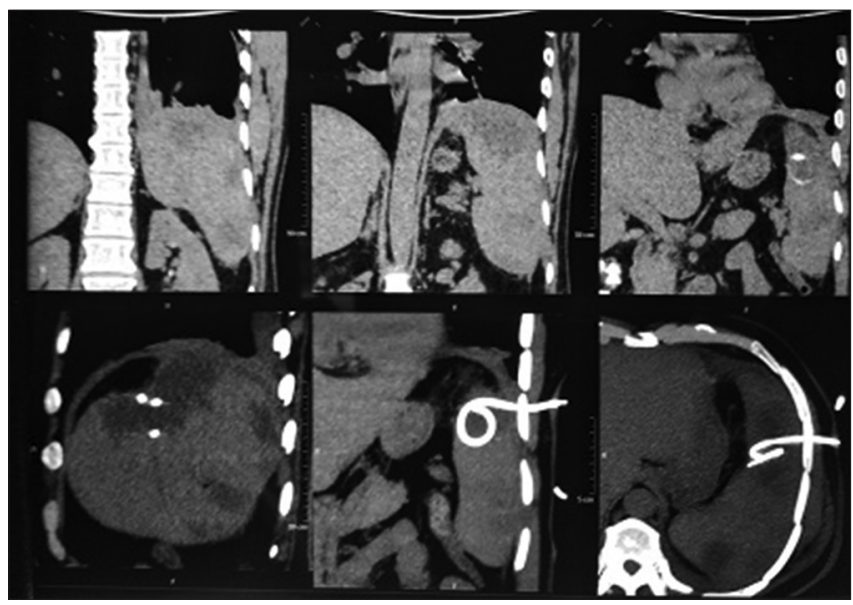

Figure 4: Contrast- enhanced computed tomography abdomen (Coronal section) after putting pigtail drainage 
insidious onset, and vague clinical presentation. ${ }^{[1,2]}$ In one study, only one case of multiple splenic abscesses due to salmonella was reported out of 68 cases of splenic abscesses. In the same study, it was also seen that spleen infected with Gram-negative bacilli was likely to develop multiple abscesses and experienced higher mortality rate than patients with Gram-positive cocci infection and solitary abscess. ${ }^{[3]}$ This case is unique in the fact that there are multiple abscesses of varying sizes since his early presentation. Though various conditions interfere with the prognosis of splenic abscess, such as underlying diseases, abscess number and size, organism spectra, and general conditions, the index patient was immunocompetent with no comorbidities. ${ }^{[4]}$ In another Indian study, 14 patients out of 18 cases had multiple abscesses, most of them having the underlying disease. Pigtail drainage was done in four patients, while splenectomy was done in six cases in this study, with one mortality each with pigtail drainage and splenectomy. ${ }^{[5]}$ The incidence of splenic abscesses in typhoid fever decreased from $2 \%$ in the preantibiotic era to less than $1 \%$ postintroduction of effective antibiotics. ${ }^{[6]}$ Diagnosis of splenic abscess with positive blood culture for salmonella typhi is rare nowadays. In a series of 16 patients of splenic abscesses, none of the cases were associated with enteric fever and other bacterial pathogens were the most common causes. ${ }^{[7]}$ The diagnosis in endemic regions like India therefore depends on a very high index of suspicion. In this particular case, the diagnosis was made on the basis of blood culture positivity with imaging evidence on USG abdomen and computed tomography. Sickle cell disease, IV drug abuse, subacute bacterial endocarditis, diabetes mellitus, HIV, and immunosuppresants are common predisposing factors. ${ }^{[8]}$ Tuberculosis should always be considered especially in the developing countries where the splenic abscess may be isolated or associated with a disseminated disease. ${ }^{[9]}$ Workup for tuberculosis was negative in this case. The treatment of splenic abscesses was splenectomy with antibiotic therapy until recently. However, over the last few years, the approach is more conservative because of better understanding of immunologic role of the spleen. Splenectomy is the preferred treatment when there is no response to antibiotics with or without percutaneous drainage. In our case, the patient has responded dramatically after draining the largest abscess and adequate course of broad-spectrum antibiotics. Percutaneous drainage is preferred due to lesser risk of intra-abdominal spillage, less complications, and better acceptance by the patient. ${ }^{[10]}$

\section{ConcLusion}

In a case of enteric fever with left upper-quadrant discomfort, a diagnosis of splenic abscess should always be considered. Patients should be given a trial of spleen-sparing strategy in the form of percutaneous drainage and antibiotics if general conditions permit.

\section{Declaration of patient consent}

The authors certify that they have obtained all appropriate patient consent forms. In the form the patient(s) has/have given his/her/their consent for his/her/their images and other clinical information to be reported in the journal. The patients understand that their names and initials will not be published and due efforts will be made to conceal their identity, but anonymity cannot be guaranteed.

\section{Financial support and sponsorship}

Nil.

\section{Conflicts of interest}

There are no conflicts of interest.

\section{RefEREnCES}

1. Allal R, Kastler B, Gangi A, Bensaid AH, Bouali O, Cherrak C, et al. Splenic abscesses in typhoid fever: US and CT studies. J Comput Assist Tomogr 1993;17:90-3.

2. Ng KK, Lee TY, Wan YL, Tan CF, Lui KW, Cheung YC, et al. Splenic abscess: Diagnosis and management. Hepatogastroenterology 2002;49:567-71.

3. Chang KC, Chuah SK, Changchien CS, Tsai TL, Lu SN, Chiu YC, et al. Clinical characteristics and prognostic factors of splenic abscess: A review of 67 cases in a single medical center of Taiwan. World J Gastroenterol 2006;12:460-4.

4. Chou YH, Tiu CM, Chiou HJ, Hsu CC, Chiang JH, Yu C, et al. Ultrasound-guided interventional procedures in splenic abscesses. Eur J Radiol 1998;28:167-70.

5. Agarwal D, Arora D, Avasthi A, Singhal S. Prospective observational study of splenic abscess (A study of 18 cases in term of clinical character, outcome and prognostic factors). CIB Tech J Surg 2015;4:17-21.

6. Bhongle NN, Nagdeo NV, Thombare VR. A splenic abscess which was caused by Salmonella typhi in a non sickler patient: A Rare case finding. J Clin Diagn Res 2013;7:537-8.

7. Ferraioli G, Brunetti E, Gulizia R, Mariani G, Marone P, Filice C, et al. Management of splenic abscess: Report on 16 cases from a single center. Int J Infect Dis 2009;13:524-30.

8. Huang DB, DuPont HL. Problem pathogens: Extra-intestinal complications of Salmonella enterica serotype typhi infection. Lancet Infect Dis 2005;5:341-8.

9. Anuradha S, Singh NP, Agarwal SK. Splenic abscess - A diversity within. J Indian Acad Clin Med 2000;1:279-81.

10. Thanos L, Dailiana T, Papaioannou G, Nikita A, Koutrouvelis H, Kelekis DA, et al. Percutaneous CT-guided drainage of splenic abscess. AJR Am J Roentgenol 2002;179:629-32. 